\title{
BANCO DE SEMILLAS, GERMINACIÓN Y LONGEVIDAD DE SEMILLAS DE RETAMA (SPARTIUM JUNCEUM, FABACEAE): IMPLICANCIAS PARA SU CONTROL
}

\author{
CRISTINA SANHUEZA ${ }^{1^{*}}$ y SERGIO ZALBA ${ }^{1}$
}

\begin{abstract}
Summary: Seed bank, germination and seed longevity of Spanish broom (Spartium junceum, Fabaceae): implications for control. The Spanish broom (Spartium junceum) is an exotic invader of natural grasslands in southern Buenos Aires mountains. We assessed the density of seeds in the soil seed bank and the percentage of germination in botanic garden experiments for samples obtained from three invaded sites. We performed essays outdoors for soil samples, and in germination chamber for seeds collected directly from plants and for seeds obtained from the soil seven years before the experiment. Seeds density in the soil ranged from 600 to 4411 seeds $/ \mathrm{m}^{2}$. Germination percentage for soil samples was $68.33 \pm 26.66 \%$. For chamber experiments, mechanical scarification lead to the germination of all the seeds in the case of those collected from the plants, compared to just $7 \pm 5.7 \%$ for the control and figures even smaller for heath shock and combination of heath shock and mechanical scarification. The experiment with seeds form the soil bank resulted in a percentage of germination of $88.73 \pm 11.16 \%$ for those seeds previously treated with mechanical scarification, $85.77 \pm 10.50 \%$ for heath shock, $40.30 \pm 10.14 \%$ for imbibitions and $43.20 \pm 12.01 \%$ for the control. Brief exposition to high temperatures, mechanical breakage of the seed cover and soil disturbances significatively favor germination and stimulate invasion.
\end{abstract}

Keywords: Spartium junceum, germination, soil seed bank, invasion, seeds longevity.

\begin{abstract}
Resumen: La retama (Spartium junceum) es una especie invasora de pastizales naturales en las sierras australes bonaerenses. Estudiamos características del proceso de germinación que resultarían clave para su control. Calculamos densidad de semillas y porcentaje de germinación para muestras de suelo provenientes de sectores invadidos. Realizamos ensayos de germinación en panes de tierra mantenidos a la intemperie y ensayos en cámara de germinación con semillas colectadas directamente del arbusto y otras colectadas del banco del suelo siete años antes del ensayo. La densidad en el banco del suelo de áreas invadidas varió entre 600 y 4.411 semillas $/ \mathrm{m}^{2}$. El porcentaje de germinación de semillas de ensayos en panes de tierra fue de $68,33 \pm 26,66 \%$. Para los ensayos en cámara, la escarificación mecánica resultó $100 \%$ de germinación para semillas recolectadas de las plantas, contra $7 \pm 5,7 \%$ para el control y valores aún inferiores para shock térmico y combinación shock térmico+escarificación mecánica El ensayo de semillas con más de siete años resultó en un porcentaje de germinación del $88,73 \pm 11,16 \%$ para la escarificación mecánica, $85,77 \pm 10,50 \%$ para shock térmico, 40,30 $\pm 10,14 \%$ para imbibición y $43,20 \pm 12,01 \%$ para el control. Altas temperaturas durante períodos cortos, lesiones en la cubierta seminal y disturbios del suelo favorecen considerablemente la germinación y estimulan los procesos de invasión.
\end{abstract}

Palabras Clave: Spartium junceum, germinación, banco de semillas, invasión, longevidad.

\section{INTRODUCCIÓN}

La persistencia de los bancos de semillas es uno de los desafíos más importantes para el control

\footnotetext{
${ }^{1}$ GEKKO - Grupo de Estudios y Conservación y Manejo - Departamento de Biología, Bioquímica y Farmacia. Universidad Nacional del Sur, San Juan N ${ }^{\circ} 670$ (8000) Bahía Blanca, Argentina.

*E-mail: ccsanhueza@yahoo.com.ar
}

exitoso de plantas exóticas invasoras. Las acciones de manejo de especies invasoras con bancos de semillas duraderos requieren la repetición de las acciones de control y una estrategia de monitoreo constante (Richardson \& Kluge, 2008; Alexander \& D`Antonio, 2003; D`Antonio \& Meyerson, 2002).

El término retama se utiliza para denominar a un conjunto de especies de la familia de las leguminosas (Fabaceae), nativas de la región del Mediterráneo y de las islas Canarias y Azores, 
reconocidas en diferentes regiones del mundo por su agresivo comportamiento invasor (Randall, 2002). Las cuatro especies más comunes son la retama escocesa (Cytisus scoparius), la retama francesa (Genista monspessulana), la retama española (Spartium junceum) y la retama portuguesa (Cytisus striatus). Generalmente han sido introducidas como plantas ornamentales, aunque también hay registros que hablan de su uso para el control de la erosión de los suelos (LeBlanc, 2001; Oneto et al., 2009).

Las especies de este grupo producen gran cantidad de semillas, un solo arbusto puede producir unas 2.000 a 3.500 chauchas. Aproximadamente el $40 \%$ de las semillas producidas germinan inmediatamente después de la dispersión, y otro $25 \%$ lo hace al año siguiente. El resto de las semillas pueden permanecer latentes en el suelo por periodos de hasta 60-80 años (Hoshovsky, 1986a; b; Bossard et al., 2000; Oneto et al., 2009, Garcia et al., 2007). La retama escocesa, por ejemplo, puede producir más de 15.000 semillas por año, en promedio menos del 5\% de las semillas se pierden por depredación de insectos, y el 98\% quedan viables (Leblanc, 2001).

Este trabajo se enfoca en poblaciones invasoras de retama española, $S$. junceum, en las sierras australes de la provincia de Buenos Aires. La retama española es un arbusto perenne y muy ramoso que puede alcanzar cuatro metros de altura. Sus raíces son muy profundas y sus hojas ovaladas permanecen poco tiempo en la planta, generalmente sólo durante los meses de septiembre a noviembre. Florece en primavera y verano con una gran profusión de flores amarillas, vistosas y de agradable aroma (Parodi, 1978). Sus semillas se encuentran en vainas o chauchas que se abren en forma violenta, expulsándolas hasta $10 \mathrm{~m}$ desde la planta madre. Las semillas son pequeñas, de entre cuatro y cinco $\mathrm{mm}$ de largo, poseen una cubierta muy dura y luego de caer pueden permanecer viables en el suelo muchos años (Hoshovsky, 1986a). Como el resto de las plantas de la familia, esta especie tiene la capacidad de fijar nitrógeno atmosférico, lo que aumenta su capacidad de alterar los procesos ecológicos de las áreas donde invade. Forma manchones muy densos que inhiben el crecimiento de cualquier otro tipo de vegetación, con frecuencia prefiere bordes de arroyos, donde su crecimiento suele ser responsable de desbordes e inundaciones. Durante el crecimiento de las plantas, los tallos más internos van muriendo, de manera que los individuos viejos retienen gran cantidad de materia seca. Esta situación, sumada a su abundancia en bordes de caminos, determina que los manchones de retama se quemen con frecuencia y que los fuegos que los afectan sean muy difíciles de controlar (Sanhueza, 2012). Los herbívoros no consumen los tejidos de las retamas, posiblemente debido a que contiene el alcaloide citisina que la vuelve tóxica (Font Quer, 1993).

La persistencia de sus semillas en el suelo, su capacidad de crecer en manchones densos, su habilidad para fijar nitrógeno y de aumentar la frecuencia e intensidad de los incendios, su resistencia a los herbívoros y su capacidad de brotar luego del corte de las porciones aéreas y del fuego, convierten a esta especie en una invasora exitosa y de muy difícil control. Además, en el caso de los pastizales pampeanos, esta especie se desarrolla en un clima similar al de su lugar de origen (Kottek et al., 2006), lo que seguramente favorece su adaptación local. La retama española posee antecedentes como invasora en distintas regiones del planeta, donde sus cualidades ornamentales hacen que se la cultive con frecuencia, pudiendo fácilmente escapar desde jardines y bordes de caminos hacia sectores silvestres. Así, por ejemplo, se ha convertido en una de las plantas invasoras más agresivas en los pastizales del sur de la provincia de Buenos Aires (Zalba \& Villamil, 2002) y de otras áreas en todo el bioma de las Pampas.

Como muchas otras fabáceas, las retamas presentan semillas duras, lo que muy posiblemente resulte en una dormición exógena. Fenner (1985) explica que las semillas con estas características tienen una dormición innata que las vuelve incapaces de germinar cuando recién han sido dispersadas, aún bajo condiciones favorables para el desarrollo y supervivencia de las plántulas. Según Nikolaeva (2001) esta incapacidad de germinar está relacionada con la cubierta externa (pericarpio y/o cubierta seminal) que resulta en una dormición física. Baskin \& Baskin (1998) atribuyen las limitaciones de germinación fundamentalmente a la impermeabilidad de la semilla. Esta dormición física puede romperse eventualmente con la abrasión o la remoción de la cubierta seminal.

Poco se sabe de la dinámica del banco de semillas de retama, de la viabilidad de las semillas 


\section{Sanhueza y S. Zalba - Semillas de retama (Spartium junceum)}

y de los factores que favorecen su germinación (Hoshovsky, 1986a; LeBlanc, 2001; Nilsen, 2000).

En pastizales invadidos por S. Junceum se han registrado bajos porcentaje de emergencia y supervivencia de plántulas de retama provenientes del banco de semillas debajo de las formaciones adultas de esta especie (Sanhueza \& Zalba, 2012).

Dilucidar los aspectos biológicos y ecológico del éxito de la retama por fuera de su rango de distribución nativo es uno de los primeros pasos que se deben transitar para comprender el proceso de invasión y resulta crucial para tomar decisiones de manejo efectivas. En particular, la identificación de los factores que rompen la dormición de las semillas permitiría anticipar procesos de reclutamiento en áreas invadidas, incluyendo la respuesta a disturbios tales como la remoción de suelo o el fuego.

Los objetivos de este trabajo son identificar factores que rompen la dormición de semillas de S. junceum, contribuir al conocimiento de la dinámica de su banco de semillas y evaluar la viabilidad de las mismas.

\section{Materiales y Métodos}

\section{Área de Estudio}

Las tareas de campo se desarrollaron en el Parque Provincial Ernesto Tornquist (PPET), la reserva natural más antigua de la provincia de Buenos Aires. El parque está ubicado en la porción central del Sistema de Ventania $\left(38^{\circ} 00^{\prime}-38^{\circ} 07^{\prime}\right.$ S y $61^{\circ} 52^{\prime}$ $62^{\circ} 03^{\prime} \mathrm{O}$ ), tiene una superficie aproximada de 6.700 ha y constituye uno de los últimos relictos protegidos de pastizal pampeano (Fig. 1).

El clima de la región es templado, con una temperatura media anual de $14^{\circ} \mathrm{C}$. En enero, el mes más cálido, la temperatura media alcanza los $20,5^{\circ} \mathrm{C}$, con una máxima absoluta de $40^{\circ} \mathrm{C}$, en cambio en el mes de julio, el más frío, la temperatura media es de $8^{\circ} \mathrm{C}$ y la mínima absoluta puede llegar a $-10^{\circ} \mathrm{C}$, con ocasionales nevadas. Los inviernos serranos son más crudos en relación con la región circundante y el riesgo de heladas se proyecta hasta la primavera avanzada (Burgos, 1968). La precipitación media

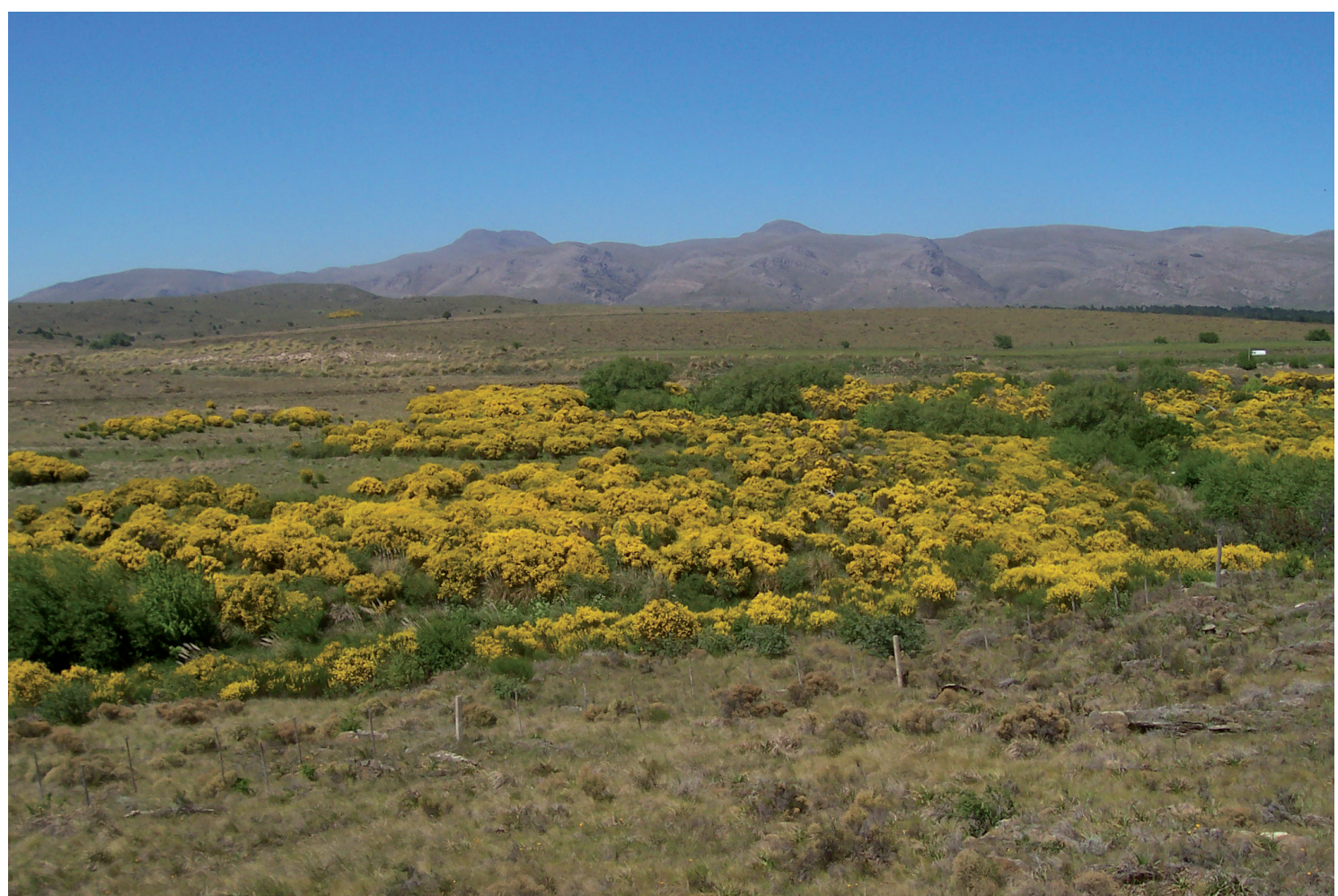

Fig.1. Pastizal de piedemonte invadido por retamas (Spartium junceum). 
anual acumulada dentro de la reserva alcanzó los $626 \mathrm{~mm}$ durante el periodo 1993-2007, según datos tomados por el personal de guardaparques.

La reserva comprende una gran diversidad de biotopos vinculada con la heterogeneidad del relieve, con el gradiente altitudinal que incluye las mayores alturas de la provincia, y con la variedad de suelos (Frangi \& Botino, 1995). Esta diversidad de ambientes se traduce en una alta riqueza de especies, resaltando el valor del área para la conservación de la biodiversidad (Kristensen \& Frangi, 1995). Quinientas cincuenta y cuatro especies vegetales (413 nativas y 141 adventicias) conforman la flora del Parque Tornquist, representando un $85 \%$ de las especies consideradas para toda la Sierra de la Ventana. De los veinte endemismos estrictos registrados en este sistema orográfico, 17 crecen dentro del Parque Tornquist (Long \& Grassini, 1997). La fauna de la reserva también incluye especies endémicas como la iguana de cobre (Pristidactylus casuhatiensis) y la culebra pintada (Lygophis elegantissimus).

\section{Estado del banco de semillas de retama}

Se seleccionaron tres sectores de pastizal de aproximadamente $15 \mathrm{~m}$ de diámetro invadidos por Spartium junceum, con una densidad promedio de siete individuos $/ \mathrm{m}^{2}$. En cada uno de ellos se tomaron doce muestras de suelo de $30 \mathrm{~cm}$ x $30 \mathrm{~cm}$ y $4 \mathrm{~cm}$ de profundidad ( $N=36$ ). Las muestras fueron trasladadas en forma de panes de tierra y colocadas en bandejas en el Jardín Botánico Pillahuincó, ubicado dentro del Parque Tornquist, tratando de provocar el menor disturbio posible en los panes. Las bandejas permanecieron a la intemperie entre los meses de febrero y abril, sujetas a las condiciones climáticas y sin agregado de agua de riego. Cada 15 días se registró el número de plántulas emergidas, las que se retiraban de las bandejas al momento de ser contadas. Al final del periodo de análisis se tamizó la tierra, registrando cuántas semillas permanecían en las muestras sin haber germinado. Se calculó la densidad de semillas en el banco contabilizando las plántulas emergidas (semillas germinadas) más las semillas no germinadas. Para cada fecha de registro y cada parcela se calculó el porcentaje de semillas germinadas (\% G).

La tasa de germinación se calculó usando la siguiente fórmula:

$\mathrm{TG}=(\mathrm{G} 1 / 1+\mathrm{G} 2 / 2+\mathrm{G} 3 / 3+\ldots+\mathrm{Gn} / \mathrm{x}) * 100 / \mathrm{S}$
Donde G1, G2, G3,.. Gn, representan, respectivamente, el número de semillas que germinaron después de 1, 2, 3, n días trascurridos, $\mathrm{x}$ es el número de días que duró el ensayo y $\mathrm{S}$ el número total de semillas en cada bandeja (López et al., 1999).

También secalculó el tiempo medio de germinación $\left(\mathrm{T}_{50}\right)$, que es el número de días transcurrido para llegar al 50\% de la germinación total obtenida al final del ensayo (Travlos et al., 2007).

\section{Factores que afectan la germinación}

Se realizaron ensayos de germinación en cámara para detectar factores que afecten la germinación. Se utilizaron para ello dos tipos de semillas: 1colectadas directamente de individuos adultos y 2colectadas desde banco de semillas del suelo.

Las semillas provenientes de plantas de retama fueron colectadas de manera aleatoria de distintos individuos durante el verano de 2004. Se utilizaron tres tratamientos pre-germinativos: escarificación mecánica, shock térmico y la combinación de ambos, a los que se sumó un control con semillas no tratadas. La escarificación mecánica consistió en raspar las semillas con una lija fina hasta lograr pequeñas hendiduras visibles en la cubierta, mientras que para el shock térmico fueron sumergidas en agua caliente $\left(100^{\circ} \mathrm{C}\right.$ temperatura inicial, dentro de un termo) durante $24 \mathrm{hs}$ y para el tratamiento combinado (escarificación mecánica+shock térmico) las semillas fueron sumergidas en agua en idénticas condiciones, previa abrasión de la cubierta seminal. El ensayo se desarrolló a $23^{\circ} \mathrm{C}$ de temperatura constante, doce horas de luz y doce de oscuridad y una humedad ambiente del $90 \%$, en cámara de germinación. Dichas condiciones fueron seleccionadas teniendo en cuenta la temperatura media de verano en la región, época en la cual la especie germina. Para cada tratamiento se colocaron cinco cajas de Petri con algodón en la base y papel de filtro por encima, sobre el cual se colocaron 20 semillas. Se registraron las semillas germinadas para cada caja cada tres días al inicio y luego cada 9-10 días, durante dos meses. Cada vez que se revisaba la cámara de germinación, las semillas germinadas se retiraban para evitar potenciales efectos inhibitorios sobre la germinación de las semillas restantes. La germinación se calculó para cada caja, en cada observación, expresada como el porcentaje de semillas germinadas ( $\% \mathrm{G})$. Las semillas fueron consideradas germinadas cuando su 


\section{Sanhueza y S. Zalba - Semillas de retama (Spartium junceum)}

radícula emergía más de $2 \mathrm{~mm}$. Se calcularon la tasa de germinación y el tiempo medio de germinación.

Las semillas provenientes del banco del suelo de áreas invadidas fueron extraídas en octubre de 2004 utilizando un tamiz y conservadas en sobres de papel en un lugar seco a temperatura ambiente hasta el ensayo en 2010. De esta forma, la edad de las semillas al momento del ensayo de germinación puede estimarse en siete años o más, puesto que el último aporte de semillas al banco del suelo antes de la colección correspondió al verano de 2003. Para estas semillas se utilizaron tres tratamientos: escarificación mecánica, imbibición y shock térmico, más un control con semillas no tratadas. La escarificación mecánica se realizó de la misma manera que en el ensayo anterior, para la imbibición las semillas se colocaron en agua a temperatura ambiente durante $24 \mathrm{hs}$ y el shock térmico se produjo sumergiendo las semillas en agua a $100^{\circ} \mathrm{C}$ durante cinco minutos. Las condiciones de la cámara de germinación fueron las mismas que para el ensayo anterior y se registraron y calcularon los mismos datos ( $\%$ G, TG y $\left.\mathrm{T}_{50}\right)$. Los porcentajes de germinación fueron analizados mediante Anova simple con el programa estadístico SPSS 7.5 y se realizaron comparaciones múltiples a posteriori siguiendo el método de Scheffé.

\section{Resultados}

\section{Estado del banco de semillas de retama}

La densidad de semillas en el banco de sectores invadidos varió entre 600 y 4.411 semillas por $\mathrm{m}^{2}$, con una densidad promedio de 1209,18 $( \pm 1029,02)$ semillas $/ \mathrm{m}^{2}$.

Las pruebas de germinación de panes de tierra de sectores invadidos resultaron en una densidad acumulada media de 811,60 plántulas $/ \mathrm{m}^{2}( \pm 722,02)$ trascurridos 90 días desde la remoción de las muestras de suelo. El pico de semillas germinadas se produjo a los 45 días. El porcentage de germinación fue del $68,33 \%( \pm 26,66)$ y el $\mathrm{T}_{50}$ de 50 días $( \pm 12,58)$ (Fig. 2$)$.

\section{Factores que afectan la germinación}

De los tratamientos pre-germinativos utilizados, el más exitoso fue el mecánico, con el que se obtuvo un $100 \%$ de germinación para el caso de semillas colectadas de individuos adultos. El pico máximo de germinación para semillas sometidas a este tratamiento

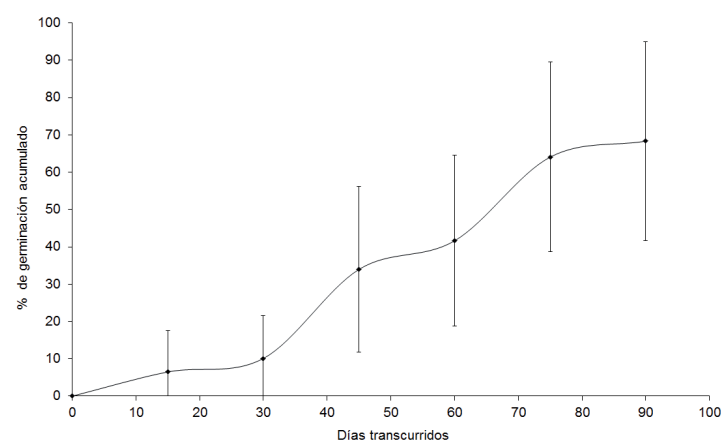

Fig. 2. Porcentaje acumulado de germinación de semillas de retama a partir de muestras provenientes del banco de semillas del suelo de áreas invadidas.

se produjo al séptimo día de colocadas en la cámara. Las semillas que recibieron escarificación mecánica + shock térmico y las de control presentaron muy bajos porcentajes de germinación (Tabla 1 y Fig. 3).

Los porcentajes de germinación promedio del ensayo con semillas recolectadas del banco del suelo mostraron diferencias significativa entre los diferentes tratamientos $(\mathrm{F}=28,778 ; \mathrm{p}<0,001)$. El mayor valor registrado correspondió a la escarificación mecánica $88,73 \%( \pm 11,16)$. Las semillas que recibieron escarificación mecánica y aquellas con shock térmico mostraron porcentajes de germinación significativamente mayores que aquellas a las se les aplicó imbibición y a las que no recibieron tratamientos pre-germinativos (contrastes de Scheffé, $P<0,001$ ) (Tabla 2 y Fig. 4).

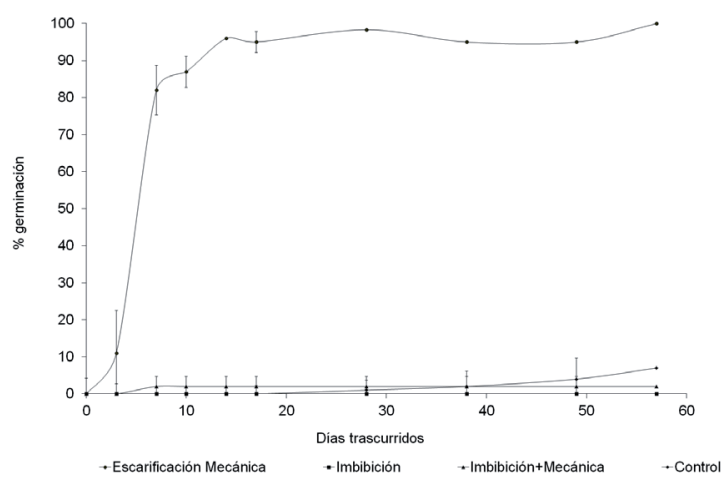

Fig. 3. Porcentaje promedio de germinación de semillas colectadas de individuos adultos de retama sujetas a distintos tratamientos pre-germinativos desde el momento de la siembra. 
Tabla 1. Porcentaje de germinación, tasa de germinación y $\mathrm{T}_{50}$ (número de días transcurridos para llegar al $50 \%$ de la germinación final) para semillas recolectadas de plantas de retama, según el tratamiento pre-germinativo aplicado.

\begin{tabular}{|lccc|}
\hline \multicolumn{1}{|c}{ Tratamiento } & $\begin{array}{c}\text { Porcentaje de germinación (\% } \\
\text { promedio } \pm \text { desvío estándar) }\end{array}$ & $\begin{array}{c}\text { Tasa de Germinación (\% } \\
\text { semillas germinadas/día) }\end{array}$ & T $_{50}$ (Días) \\
\hline Escarificación mecánica & $100 \pm 0$ & $37,6 \pm 0,31$ & $0 \pm 0$ \\
Shock térmico & $0 \pm 0$ & $0 \pm 0$ & $37 \pm 27,38$ \\
Shock térmico + & $2 \pm 2,7$ & $0,94 \pm 5,6$ & 0 \\
Escarificación mecánica & $7 \pm 5,7$ & $0,29 \pm 0,31$ & $47,8 \pm 9,52$ \\
\hline Control & & & \\
\hline
\end{tabular}

\section{Discusión}

La densidad de semillas de S. junceum en el suelo de sectores invadidos en pastizales de las sierras australes de Buenos Aires alcanzó valores tan altos como aquellos reportados para áreas invadidas por especies de otros géneros del grupo de las retamas, como Cytisus y Genista (Sheppard \& Hosking, 2000; Alexander \& D`Antonio, 2003; García et al., 2007).

Numerosos autores reportan un banco de semillas persistente para la retama (Hoshovsky, 1986a; Bossard et al., 2000; LeBlanc, 2001; DiTomaso \& Healy, 2007; Oneto et al., 2009), pero

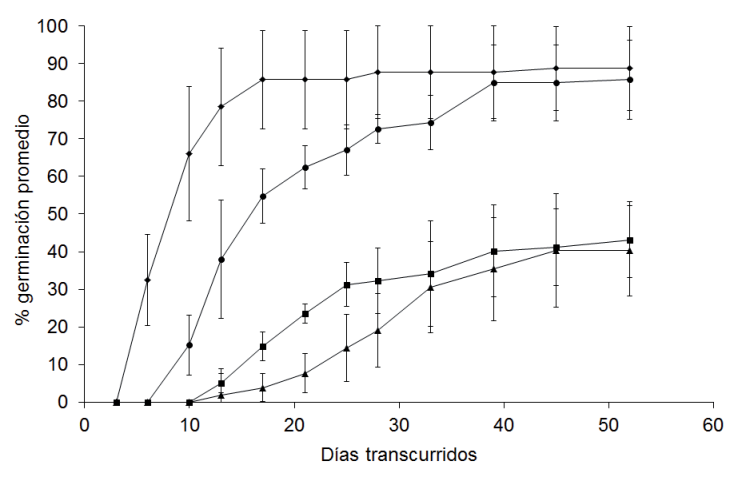

$\rightarrow$ Esc. Mecánica -Shock Térmico

-Imbibición

- Control

Fig. 4. Curvas de germinación para semillas de retama con más de siete años de edad tomadas del banco de semillas del suelo y expuestas a diferentes tratamientos pre-germinativos. no existen hasta el momento registros precisos de la longevidad de sus semillas. Nilsen (2000) propone, que las semillas de $S$. junceum permanecerían viables al menos por cinco años, sin citar los datos en los que se sustenta esta afirmación. Nuestro trabajo extiende de manera experimental dicha estimación hasta un mínimo de siete años, con valores promedio de capacidad germinativa que superan el $80 \%$, lo que hace suponer que la viabilidad de las semillas podría extenderse mucho más.

Los métodos para emular en condiciones de laboratorio la acción natural de diferentes agentes que estimulan la germinación, como la degradación de la cubierta seminal por parte de la flora microbiana del suelo, los incendios naturales u otros disturbios, resultan clave para entender la biología reproductiva de una especie y para comprender de qué depende su eventual establecimiento e invasión. Sin embargo, las conclusiones elaboradas a partir de estos ensayos deben ser tomadas con cautela, debido a la simplificación que implican respecto de las condiciones a campo. Según los resultados de los ensayos de germinación realizados en este trabajo, la escarificación mecánica juega un papel muy importante en la ruptura de la dormición de las semillas de la retama. Al comparar los porcentajes de germinación obtenidos para el control y para el tratamiento de escarificación mecánica, se evidencia claramente el efecto inhibitorio de la cubierta seminal, situación muy común en las leguminosas (López et al., 1999). El porcentaje de germinación alcanzado en asociación con la escarificación 


\section{Sanhueza y S. Zalba - Semillas de retama (Spartium junceum)}

mecánica excede los valores reportados para diversos tratamientos pre-germinativos en otros trabajos, a excepción de algunos ensayos de semillas remojadas en acido sulfúrico durante 60 y 90 minutos (López et al., 1999) que resultaron también en un $100 \%$ de germinación. Aún así, las tasas de germinación en el trabajo citado, fueron más bajas que las alcanzadas al aplicar escarificación mecánica a las semillas de retamas del Parque Tornquist $(7,72 \%$ para 60 minutos y $21,80 \%$ para 90 minutos en ácido, contra $37,6 \%$ en nuestros resultados). La tasa de germinación es un parámetro muy importante desde el punto de vista práctico, porque tiene una relación más directa que el porcentaje de germinación respecto de las posibilidades reales de establecimiento de las plántulas. Una tasa de germinación alta puede resultar en una mayor capacidad de colonización, sobre todo en especies que se ven favorecidas por los disturbios, como es el caso de la mayoría de las plantas exóticas invasoras. La escarificación química resultaría clave para promover la germinación de las semillas, según los resultados de López y colaboradores (1999). Sin embargo, la citotoxicidad de las semillas y la inexistencia de registros de consumo por parte de la fauna (Barboni et al., 1994; Hoshovsky, 1986a), sumados a los resultados de este trabajo indicarían que en el ambiente natural la escarificación mecánica y los agentes que pudieran promoverla resultarían de mayor importancia para el proceso de invasión, posiblemente en combinación con procesos químicos y microbiológicos del suelo.

Desde hace tiempo se reconoce que las perturbaciones facilitan las invasiones y este principio o variaciones del mismo, son quizás uno de los tópicos más aceptados en el campo de la ecología de las invasiones (Hobbs, 1989; Lockwood et al., 2007). Una perturbación, por su propia naturaleza, crea ventanas de invasión que favorecen el avance de las especies exóticas. Los disturbios pueden crear espacios vacíos, liberar recursos limitantes y/o alterar las interacciones, por lo que se los considera a menudo como precursores necesarios para el establecimiento de muchas especies exóticas (Rejmánek, 1989; Mack, 1989; Hobbs 1989). En el caso de las retamas, los disturbios podrían, además, promover la pérdida de dormición externa de las semillas, combinándose así condiciones que resultarían muy propicias para promover la invasión.
Tabla 2. Tasa de germinación, porcentaje de germinación y $\mathrm{T}_{50}$ para semillas de retama de más de siete años de edad tomadas de muestras de suelo y expuestas a diferentes tratamientos pregerminativos. Letras distintas indican diferencias significativas entre tratamientos (contrastes de Scheffé, $P<0,001$ ).

\begin{tabular}{|c|c|c|c|}
\hline $\begin{array}{c}\text { Tratamiento } \\
\text { pre-germinativo }\end{array}$ & $\begin{array}{c}\text { Tasa de } \\
\text { germinación } \\
\text { (\%sem/ día) } \\
\text { ( } \pm \text { DE) }\end{array}$ & $\begin{array}{c}\% \text { de } \\
\text { germinación } \\
( \pm D E)\end{array}$ & $\mathbf{T}_{50}$ \\
\hline $\begin{array}{l}\text { Escarificación } \\
\text { mecánica }\end{array}$ & $27,44( \pm 9,61)$ & $88,73( \pm 11,16) a$ & 8,4 \\
\hline Shock térmico & $19,79( \pm 3,73)$ & $85,77( \pm 10,50) a$ & 14,6 \\
\hline Imbibición & $5,49( \pm 1,81)$ & $40,30( \pm 10,14) b$ & 28,4 \\
\hline $\begin{array}{l}\text { Control (sin } \\
\text { tratamiento) }\end{array}$ & $8,59( \pm 2,15)$ & $43,2( \pm 12,01) b$ & 20,2 \\
\hline
\end{tabular}

El elevado porcentaje de germinación $(68 \% \pm$ $26,66)$ que se observó en el ensayo de germinación a partir de muestras de suelo refuerza la hipótesis que señala la importancia de los disturbios sobre el establecimiento de la retama. La población muestra un alto porcentaje de viabilidad entre las semillas almacenadas en el suelo, pero bajos valores de germinación en situaciones de ausencia de disturbio (manchones de retama que no han sido afectados por incendios ni por agentes de remoción de suelo). Muestreos de reclutamiento desarrollados en sectores invadidos sin disturbio aparente resultaron en una densidad de 0,57 plántulas $/ \mathrm{m}^{2}$ (Sanhueza, 2012), mientras que la remoción del suelo realizada en los ensayos de germinación en este trabajo multiplicó esos valores en casi mil quinientas veces (811 plántulas $/ \mathrm{m}^{2}$ ). La causa próxima de esta diferencia podría estar relacionada con un incremento en la escarificación a partir de la remoción del suelo, aunque como se aclaró anteriormente este proceso se realizó cuidando de minimizar el impacto sobre el sustrato removido. De manera alternativa o complementaria, el movimiento del suelo podría haber resultado en cambios en las condiciones hídricas, en la temperatura, la insolación o en procesos químicos o microbiológicos asociados con el hecho de mover las muestras fuera de la cobertura densa de los adultos. Los valores de $\mathrm{T}_{50}(=50) \mathrm{y}$ de tasa de germinación $(\mathrm{TG}=3,83)$ obtenidos en 
este ensayo fueron menores que en el caso de la escarificación realizada en laboratorio, sin embargo esto podría explicarse por los efectos más intensos de la abrasión manual. Al observar el porcentaje de germinación de semillas cosechadas directamente de las plantas $(7 \%)$ y de aquellas provenientes de un banco del suelo con una antigüedad mínima de siete años $(43,2 \%)$, se podría reforzar la idea de un lento desgaste de la cubierta seminal en el suelo.

De manera similar, el fuego podría ser un factor clave en el proceso de invasión de retamas en la Sierra de la Ventana, considerando el posible efecto de la temperatura como agente de ruptura de la dormición de las semillas. Exciten trabajos (Travlos et al., 2007; Travlos 2009; López et al., 1999; Hanley, 2009; Reyes \& Trabaud, 2009) que realizaron ensayos en laboratorio simulando el aumento de temperatura en el suelo asociado con un incendio. Sus resultados demostraron la importancia de este factor para la germinación de la retama. Nuestros ensayos no evidenciaron una relación clara entre exposición al calor y porcentaje de germinación. Mientras que las semillas colectadas de plantas adultas colocadas en agua caliente por 24 hs no mostraron grandes cambios en sus tasas de germinación, aquellas recogidas del suelo y expuestas a altas temperaturas durante sólo cinco minutos sí incrementaron su respuesta germinativa. Una posible explicación para estas diferencias podría ser que los embriones de las semillas del primer ensayo hubieran sufrido daños o muerte, asociados a la combinación de tiempos prolongados a altas temperaturas (Hanley, 2009; Reyes \& Trabaud, 2009). Otra posible explicación podría ser el desarrollo de hongos que eventualmente inhibieran la germinación como consecuencia de la humedad retenida por las semillas remojadas durante 24 hs.

\section{Conclusiones}

Este trabajo reporta el primer dato conocido de viabilidad para semillas de Spartiun juncem: más de siete años, almacenadas en laboratorio. El alto porcentaje de germinación de las semillas con una edad superior a siete años sugeriría que su viabilidad puede ser aún mayor, resaltando las dificultades para controlar esta especie una vez establecida en un área.

La escarificación mecánica resulta un factor clave en la promoción de la germinación de retama. Los agentes que provocan disturbios en el suelo, u otros asociados con la eliminación de la cubierta vegetal, podrían desencadenar la germinación masiva de la especie y su posterior avance e invasión. Por esta razón se debe prestar particular atención a aquellos eventos que provoquen disturbios en el suelo, concentrando en los sitios afectados las tareas de monitoreo y control precoz, así como evitar dichos disturbios en áreas prioritarias de conservación expuestas a la llegada de semillas de esta especie.

\section{Agradecimientos}

Agradecemos al Consejo Nacional de Investigaciones Científicas y Técnicas (CONICET) y a la Universidad Nacional del Sur que financiaron este trabajo. La Dirección de Áreas Naturales Protegidas de Buenos Aires que prestó apoyo a las tareas de campo.

\section{Bibliografía}

ALEXANDER, J. A. \& C. M. D'ANTONIO. 2003. Effectiveness of different control methods on the removal of French and Scotch Broom and restoration of grassland in coastal California. Ecol. Rest. 21: 191-198.

BARBONI, L., A. MANZI, B. BELLOMARIA \& A. M. QUINTO. 1994. Alkaloid content four Spartium junceum populations as a defensive strategy against predators. Phytochemistry 37: 1197-1200.

BASKIN, C. \& J. M. BASKIN. 1998. Seeds. Ecology, Biogeography and Evolution of Dormancy and Germination. Academic Press, California, USA.

BOSSARD, C., J. M. RANDALL \& M. C. HOSHOVSKY. 2000. Invasive Plants of California's Wildlands. University of California Press, Berkeley.

BURGOS, J. 1968. El clima de la provincia de Buenos Aires en relación con la vegetación natural y el suelo. In: CABRERA, A. L. (ed.), Flora de la provincia de Buenos Aires, pp. 33-100. Colección Científica INTA, Buenos Aires.

D'ANTONIO, C. \& L. A. MEYERSON. 2002. Exotic plant species as problems and solutions in ecological restoration: a synthesis. Restor. Ecol. 10: 703-713.

DITOMASO, J. M. \& E. A. HEALY. 2007. Weeds of California and other western states. University of California Agriculture and Natural Resources, Publication 3488, California. 


\section{Sanhueza y S. Zalba - Semillas de retama (Spartium junceum)}

FENNER, M. 1985. Seed Ecology. Chapman and Hall Ed., London, New York.

FONT QUER, P. 1993. Plantas medicinales el dioscórides renovado. Editorial Labor, Barcelona.

FRANGI, J. L. \& O. J. BOTINO. 1995. Comunidades vegetales de la Sierra de la Ventana, Provincia de Buenos Aires, Argentina. Rev. Fac. Agron. La Plata 71: 93-133.

GARCÍA, R. A., A. PAUCHARD \& E. PEÑA. 2007. Banco de semillas, regeneración y crecimiento de Teline monspessulana (L.) K. Koch después de un incendio forestal. Gayana Bot. 64: 201-210.

HANLEY, M. E. 2009. Thermal shock and germination in North-West European Genisteae: Implications for heathland management and invasive weed control using fire. Appl. Veg. Sci. 12: 385-390.

HOBBS, R. J. 1989. The nature and effects of disturbance relative to invasions. In: DRAKE J. A., H. A. MOONEY, D. F. DI CASTRI, R. H. GROVES, F. J. KRUGER, M. REJMANEK \& M. WILLIAMSON (eds.), Biological Invasions A Global Perspective, pp. 369-405. Scientific Committee on Problems of the Environment (SCOPE), New York.

HOSHOVSKY, M. 1986 a. Spartium junceum (Spanish broom). The Nature Conservancy - Element Stewardship Abstract. The Nature Conservancy, Arlington, VA.

HOSHOVSKY, M. 1986 b. Cytisus scoparius and Genista monspessulanus (Scotch Broom, French Broom). The Nature Conservancy - Element Stewardship Abstract. The Nature Conservancy, Arlington, VA.

KRISTENSEN, M. J. \& J. L. FRANGI. 1995. La Sierra de la Ventana: una isla de biodiversidad. Ciencia Hoy 5: 25-34.

KOTTEK, M., J. GRIESER, C. BECK, B. RUDOLF \& F. RUBEL. 2006. World Map of the Köppen-Geiger climate classification updated. Meteorol. Z. 15: 259263.

LEBLANC, J. W. 2001. Getting a Handle on Broom. Publication 8049. University of California Agriculture and Natural Resources, Oakland.

LOCKWOOD, J. L., M. F. HOOPES \& M. P. MARCHETTI. 2007. Invasion Ecology. Blackwell Publishing, Oxford.

LONG, M. A. \& C. GRASSINI. 1997. Actualización del conocimiento florístico del Parque Provincial Ernesto Tornquist. Ministerio de Asuntos Agrarios de la provincia de Buenos Aires y Universidad Nacional del Sur, Bahía Blanca.

LÓPEZ, J., J. A. DEVESA, T. RUIZ \& A. ORTEGAOLIVENCIA. 1999. Seed germination in Genisteae (Fabaceae) from South-West Spain. Phyton-Ann. Rei. Bot. A. 39: 107-128.

MACK, R. M. 1989. Temperate grasslands vulnerable to plant invasions: characteristics and consequences. In:
DRAKE, J. A., H. A. MOONEY, D. F. DI CASTRI, R. H. GROVES, F. J. KRUGER, M. REJMANEK \& M. WILLIAMSON (eds.), Biological Invasions A Global Perspective, pp. 155-179. Scientific Committee on Problems of the Environment (SCOPE), John Wiley \& Sons, Chichester.

NIKOLAEVA, M. G. 2001. An update of Nikolaeva's seed dormancy classification system and its relevance to the ecology, physiology, biogeography and phylogenetic relationships of seed dormancy and germination. Modified from an article published in Russian in Bot. Zhurn. 86: 1-14.

NILSEN, E. T. 2000. Spartium junceum L. In: BOSSARD, C., J. M. RANDALL \& M. C. HOSHOVSKY (eds.), 2000. Invasive Plants of California's Wildlands, pp. 306-309. University of California Press, London.

ONETO, S. R., J. M. DITOMASO \& G. B. KYSER. 2009. Brooms: Integrated Pest Management for Home Gardeners and Landscape Professionals. In: Flint M. L \& M. L. Fayard (eds.), Pest notes, Publication 74147. University of California Statewide, Integrated Pest Management Program, Agriculture and Natural Resources, Davis.

PARODI, L. R. 1978. Enciclopedia Argentina de Agricultura y Jardinería. Descripción de las plantas cultivadas. Tomo I. ACME SACI, Buenos Aires.

RANDALL, R. P. 2002. A global compendium of weeds. R.G and Richardson, Melbourne.

REJMÁNEK, M. 1989. Invasibility of Plant Communities. In: DRAKE, J. A., H. A. MOONEY, D. F. DI CASTRI, R. H. GROVES, F. J. KRUGER, M. REJMANEK \& M. WILLIAMSON (eds.), Biological Invasions: A Global Perspective, pp. 369-388. Scientific Committee on Problems of the Environment (SCOPE), New York.

REYES, O \& L. TRABAUD. 2009. Germination behaviour of 14 Mediterranean species in relation to fire factors: Smoke and heat. Plant Ecol. 202:113121.

RICHARDSON, D. M. \& R. L. KLUGE. 2008. Seed banks of invasive Australian Acacia species in South Africa: Role in invasiveness and options for management. Perspect. Plant. Ecol. 10: 161-177.

SANHUEZA, C. 2012. Ecología y manejo de leguminosas invasoras en la Sierra de la Ventana. Tesis doctoral. Universidad Nacional del Sur. Bahía Blanca, Buenos Aires Argentina.

SANHUEZA, C \& S. M. ZALBA. 2012. Experimental control of Spanish broom (Spartium junceum) invading natural grasslands. Manage. Biol. Invasions 3: 97-104.

SHEPPARD, A. W. \& J. R. HOSKING. 2000. Broom management. Plant Protec. Quart. 15: 134-186.

TRAVLOS, I. S. 2009. Seed germination of several invasive species potentially useful for biomass 
Bol. Soc. Argent. Bot. 49 (1) 2014

production or revegetation purposes under semiarid conditions. Acta Biol. Cracov. Bot. 51: 35-37.

TRAVLOS, I. S., G. ECONOMOU \& A. J. KARAMANOS. 2007. Seed germination and seedling emergence of Spartium junceum L. in response to heat and other pre-sowing treatments. $J$. Agron. 6: 152-156.
ZALBA, S. \& C. B. VILLAMIL. 2002. Woody plant invasions in relictual grasslands. Biol. Invasions 4: 55-72.

Recibido el 8 de mayo de 2013, aceptado el 26 de septiembre de 2013. 